Check for updates

Cite this: RSC Adv., 2017, 7, 48054

Received 14th July 2017

Accepted 5th October 2017

DOI: 10.1039/c7ra07739d

rsc.li/rsc-advances

\title{
Simultaneous enhancements in the strength, modulus and toughness of electrospun polymeric membranes $\uparrow$
}

\author{
Yiyang $\mathrm{Xu}$, ${ }^{\mathrm{ab}}$ Xuebing Zhang, ${ }^{\mathrm{b}}$ Xuan Wang, ${ }^{\mathrm{b}}$ Xuyan Li, ${ }^{\mathrm{b}}$ Changyu Shen, ${ }^{\mathrm{b}}$ \\ Xiaofeng Wang (DD *abc and Qian Li ${ }^{\star a b}$
}

\begin{abstract}
We have developed a self-reinforcement method, referred to as the solvent-non-solvent method, which optimized the mechanical performance of electrospun (ES) polymeric membranes. It was found that an as-spun polycarbonate (PC) membrane post-treated (immersion) by a mixed solvent composed of $30 \%$ solvent and $70 \%$ non-solvent achieved simultaneous enhancements in the strength (128\%), modulus (296\%) and strain at break (88\%). Structural investigations and comparisons enabled us to attribute these enhancements to the fusion of junction points between fibers. Furthermore, a post-treated ES polylactic acid (PLA) membrane for tissue engineering exhibited not only enhanced mechanical properties but also improved cell viability and proliferation.
\end{abstract}

\section{Introduction}

In 21st century technology, electrospun (ES) polymeric membranes are widely employed in biomedicine, tissue engineering, lithium batteries, and air filtration owing to their highly porous structure and huge specific surface areas. ${ }^{1-5}$ Among all the factors that have a major influence on the applications of ES nanofibrous membranes (ENMs), their mechanical properties are regarded as more important, in particular in the fields of biological applications and filtration. ${ }^{6-8}$ Although ES nanofibers exhibit excellent strength due to their highly oriented chains along the fiber axis, ${ }^{9-12}$ ENMs display poor mechanical performance ${ }^{13-16}$ because of limited contact between the fiber surfaces and low contact strength..$^{17,18}$ Determining how to improve the mechanical properties of ENMs would be of great value in promoting their applications.

There are several reported methods of improving the strength of ENMs, which roughly fall into three categories. Some studies demonstrated the enhancement of ES nanofibers by adding nanomaterials with superior strength, such as carbon nanotubes and graphene, to the polymer solution in the process of electrospinning. ${ }^{19,20}$ However, the cost was very high and in some cases the method was ineffective. ${ }^{21}$ Another method is

${ }^{a}$ National Center for International Research of Micro-Nano Molding Technology, Key Laboratory for Micro Molding Technology of Henan Province, Zhengzhou University, Zhengzhou 450001, China. E-mail: xiaofengwang@zzu.edu.cn; qianli@zzu.edu.cn

${ }^{b}$ School of Mechanics \& Engineering Science, Zhengzhou University, Zhengzhou 450001, China

'State Key Laboratory of Molecular Engineering of Polymers, Fudan University, Shanghai 200433, China

$\dagger$ Electronic supplementary information (ESI) available. See DOI: 10.1039/c7ra07739d thermal treatment, which is an effective way to strengthen ES membranes but needs accurate control of temperature and/or pressure. ${ }^{7,8,22}$ If these are not precisely controlled, the porous structure of the ES membrane might be destroyed, which would make it less suitable for applications. ${ }^{23}$ The third method attempts to reinforce ENMs by blending them with other polymers ${ }^{13}$ which is easily carried out but is greatly limited by the choice of materials, because there are few polymers that could be simultaneously dissolved by the same solvent.

Here, we present a novel method, referred to as the solventnon-solvent method, of structurally reinforcing ES membranes (polycarbonate [PC] was selected as an example) by enhancing the contact strength between the fiber surfaces, which was achieved by fusing the junction points of ES nanofibers. Firstly, we adjusted the solvent/non-solvent ratio to swell the surfaces of the fibers by immersion. As the swollen surfaces of different fibers contacted each other, molecular chains of the swollen fiber surfaces underwent entanglement, which led to fusion of the contacting fiber surfaces after complete evaporation of the solvent and non-solvent. Fusion of the fiber surfaces could significantly enhance the mechanical performance of the asspun membrane.

\section{Experimental}

The PC membrane was electrospun from a solution of $16 \%(\mathrm{w} /$ w) PC using a mixed solvent of tetrahydrofuran (THF) and dimethylformamide (DMF) in a ratio of $7: 3(\mathrm{v} / \mathrm{v})$. Reported ES PC fibers have an average diameter of approximately $2 \mu \mathrm{m},{ }^{24,25}$ which will greatly limit their application. To reduce the diameter of the PC fibers, hexadecyltrimethylammonium bromide (CTAB) was added to a solution with a content of $0.2 \%(w / w) ~ P C$, 

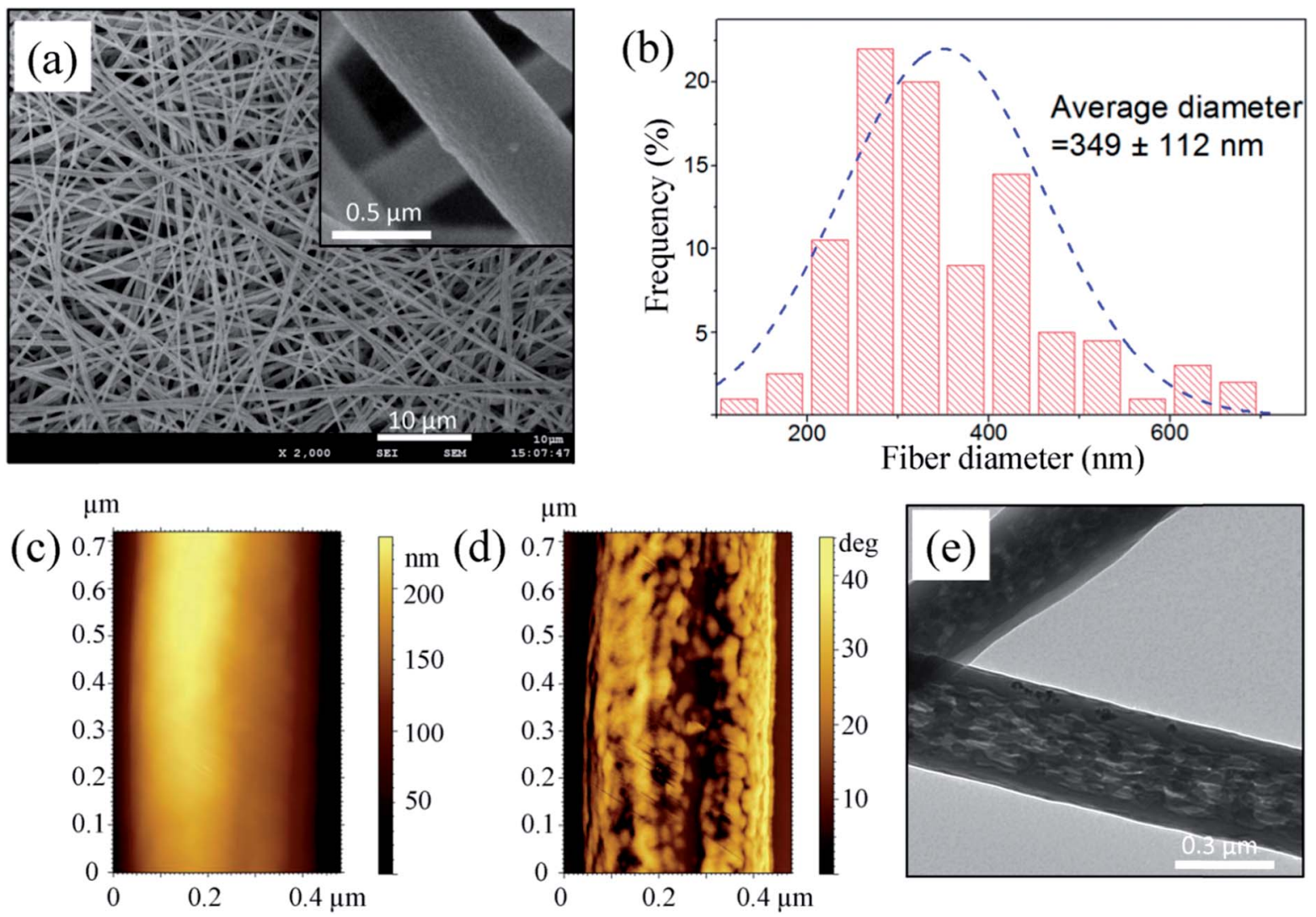

Fig. 1 Morphology of as-spun PC fibers: (a) scanning electron microscopy images, (b) corresponding fiber diameter distribution, (c) topography, (d) phase contrast image, and (e) TEM image.

which led to a sharp increase in the conductivity and a decrease in the viscosity of the solution (shown in Fig. S1 $\dagger$ ). After this adjustment, the diameter of the ES PC fibers decreased to $349 \pm$ $112 \mathrm{~nm}$ and the fiber surfaces were smooth, as shown in Fig. 1a and $b$. More experimental details are provided in the ESI. $\dagger$

\section{Results and discussion}

The topography and phase contrast were imaged using atomic force microscopy and substructures within the PC nanofibers were imaged using transmission electron microscopy (TEM), as shown in Fig. 1c-e. The topography of a single PC nanofiber displayed a smooth surface; however, a phase contrast image of the same fiber exhibited a surface composed of highly aligned irregular fibrils. A TEM image also revealed such structures inside the PC nanofibers, which might be caused by high elongation of the charged jet and fast solvent evaporation. ${ }^{9}$ As we previously measured, ${ }^{26}$ owing to their highly oriented chain structure the strength of ES PC nanofibers could be as high as $780 \mathrm{MPa}$ and exceed those of common metals such as iron and copper.

As discussed above, ES polymeric nanofibers exhibit superior mechanical properties; however, those of ES polymeric membranes are very poor, because the strength of an ES membrane is impaired by limited contact and low contact strength between the fiber surfaces. ${ }^{17,18}$ The most effective way to reinforce an ES membrane is to improve the contact strength between the fiber surfaces. ${ }^{27}$ Huang et al. increased the strength of an ES membrane using solvent vapor, which facilitated fusion between fibers at their junction points; ${ }^{17}$ however, it took several hours for the junction points to swell and fuse. Here, we suppose that water (non-solvent) can decrease the solubility of PC in THF (solvent) and there must be a critical ratio of water to THF, which makes the mixed solvent quickly swell, but not dissolve, PC fibers. Immersed in such a solvent, chains at swollen junction points may become entangled with each other and, after complete evaporation of the mixed solvent, the strength of the junction points will be greatly improved.

To test this hypothesis, the ratio of water to THF was set at values of from $5: 5$ to $9: 1$, and as-spun PC membranes were immersed in the respective mixed solvents for $0.5 \mathrm{~h}$. After immersion, all the samples were transferred to a ventilated area to allow the mixed solvent to evaporate. The macro-scale appearance of the post-treated membranes is shown in Fig. 2a, with red dashed circles marking the treated areas. There were obvious signs of destruction of the membrane, which was caused by dissolution of PC fibers, at ratios of $5: 5$ and $6: 4$. As the ratio was increased to values greater than or equal to $7: 3$, all the samples remained undestroyed. The micro-scale appearance of the post-treated membranes at ratios ranging from $5: 5$ to $9: 1$ is shown in Fig. $2 b-f$, respectively. As seen in Fig. $2 \mathrm{~b}$ and $\mathrm{c}$, the fibers were clearly dissolved and nearly all 

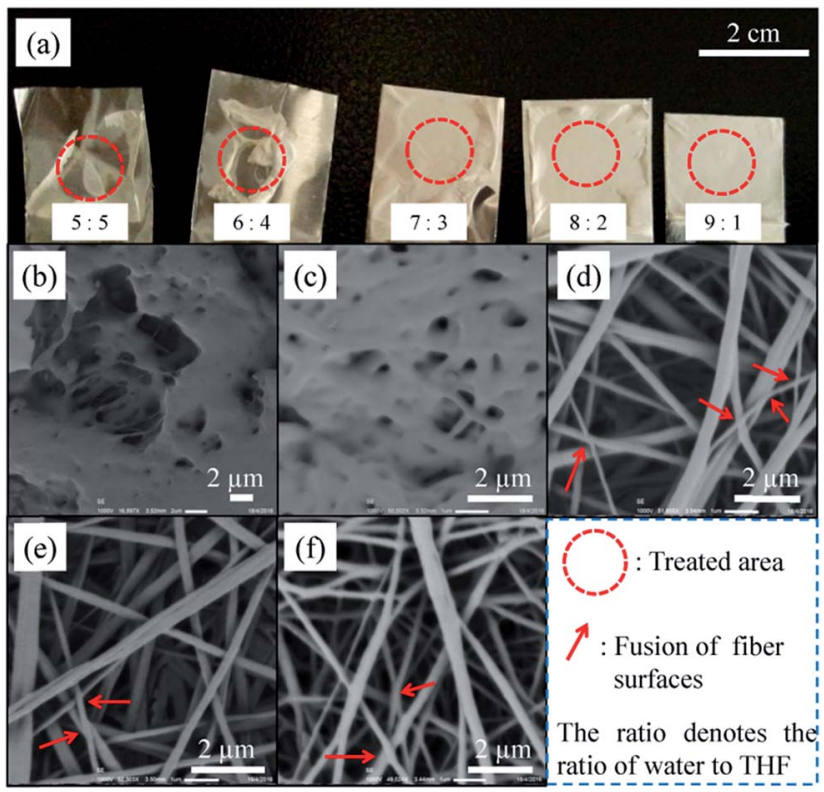

Fig. 2 (a) Macro-scale appearance and (b-f) micro-scale appearance of post-treated membranes at water/THF ratios ranging from $5: 5$ to $9: 1$.

their porous structure was destroyed, which greatly impaired the application of the membranes. As the ratio was increased to greater than $6: 4$, the morphology and porous structure of the fibers were well maintained. Furthermore, clear fusion of fiber surfaces occurred at certain points, which are marked with red arrows in each image, and more fusion of fibers occurred at a ratio of $7: 3$; accordingly, $7: 3$ might be the critical ratio in this case.

The mechanical properties of treated and untreated membranes are shown in Fig. 3. Water/THF ratios of $5: 5$ and $6: 4$ were not used, because the porous structure of the membrane was seriously damaged at these two ratios, which made the membrane less useful. As shown in Fig. 3a, the elastic stages for the treated and untreated samples were all found to occur around a strain of $8 \%$, which is the same as the value for bulk PC. ${ }^{28}$ The post-treated membrane exhibited an increase in the modulus and strength in proportion to the content of THF. This is because mixed solvents with higher contents of THF had a higher chance of swelling the fibers, which led to more fusion of junction points (see Fig. 2d-f). Before fusion, the strength of junction points was influenced by intermolecular forces (i.e., van der Waals forces), and, after fusion, they were greatly improved because of chains entanglement which led to enhanced interaction between molecular chains.

After the yield point, all the membranes exhibited strain hardening behavior; however, the respective ranges were very different. The as-spun membranes treated with mixed solvents with an increased THF content displayed an enlarged strain hardening range, which was also in proportion to the THF content. Judging from the appearance of fractures in the original membrane (shown in Fig. S2a $\dagger$ ), the breaks in the membrane might be related to slippage between fibers. After (a)

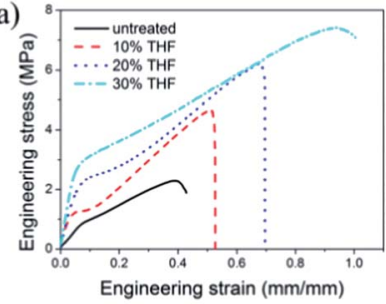

(c)

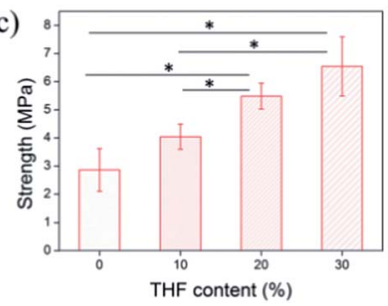

(b)
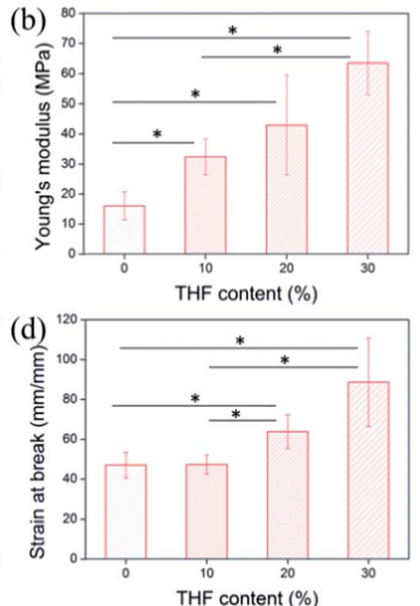

Fig. 3 (a) Strain-stress curve, (b) Young's modulus, (c) tensile strength, and (d) strain at break of PC membranes treated by mixed solvents with varying THF contents. * denotes $p<0.05$, which indicates that the two groups of data used for comparison were statistically and significantly different.

the yield point, the junction points between fibers could not bear an additional applied load, which inevitably led to the disassembly of junction points and slippage of fibers. As both disassembly and slippage increased to a certain degree, the membrane would fracture. However, the fused fiber surfaces effectively resisted disassembly and slippage (see Fig. S2b $\dagger$ ), which led to a larger strain hardening range and contributed to an increase in strain at break. The statistical results (Fig. 3b-d) showed that for the membrane post-treated with the mixed solvent with the critical ratio (water $: \mathrm{THF}=7: 3$ ) the Young's modulus, strength, and strain at break simultaneously increased by $298 \%, 128 \%$, and $88 \%$, respectively. The symbol * in Fig. $3 \mathrm{~b}-\mathrm{d}$ represents a value of $p<0.05$, which indicates that the two groups of data are significantly different and fully demonstrates the effectiveness of this method.

(a)
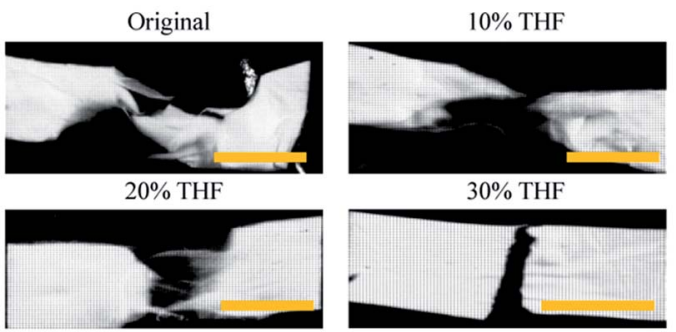

(b)

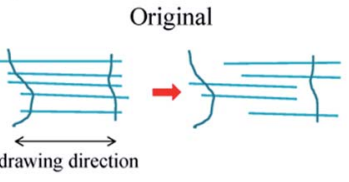

Treated by mixed solvent containing $30 \% \mathrm{THF}$

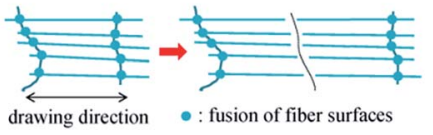

Fig. 4 (a) Macro-scale appearance of treated and original membranes at break and (b) possible mechanism of this variation. The scale bar represents $5 \mathrm{~mm}$. 
Interestingly, as shown in Fig. 4a, the membrane with a brittle fracture appearance (treated by the mixed solvent with $30 \%$ THF) exhibited greater toughness; however, the membrane with a ductile fracture appearance (original) was less tough. A possible mechanism of this uncommon phenomenon is that fusion of the fiber surfaces effectively restrained fiber slippage and led to neater fractures (see Fig. 4b), which also explains the increase in toughness after treatment. Generally speaking, the strength of materials is enhanced at the expense of their toughness and vice versa. However, the method that we proposed improved effectively and simultaneously the strength, modulus, and toughness of the ES nanofibrous PC membrane. Moreover, we tested the availability of this method for ES PLA membrane for tissue engineering. It turned out that besides mechanical performance ( $c f$. Fig. S3†), post-treated ES PLA membrane also revealed improvement of cell viability and proliferation of human umbilical vein endothelium cells (HUVECs, Oligobio, Beijing, China) (shown in Fig. S4†).

\section{Conclusions}

In summary, ES PC membranes exhibited poor mechanical properties because of the limited number of junction points and low contact strength of the contacting fiber surfaces. By our solvent-non-solvent method, the mechanical performance was significantly improved owing to the fusion of junction points, which was caused by swelling of fiber surfaces. A water/THF ratio of $7: 3$ might be the critical ratio; after being treated by a mixed solvent with this ratio an ES PC membrane displayed substantial and simultaneous increases in modulus (296\%), strength $(128 \%)$, and strain at break $(88 \%)$. This method also promotes biocompatibility improvement of ES tissue engineering scaffold.

\section{Conflicts of interest}

There are no conflicts to declare.

\section{Acknowledgements}

The authors acknowledge financial support from the International Science \& Technology Cooperation Program of China (2015DFA30550), the National Natural Science Foundation of China (11372286), the Key Project of Science and Technology of the Education Department of Henan Province (15A430047), and the Key Project of International Cooperation of Henan Province (152102410013).

\section{Notes and references}

1 S. L. Duque, N. Brack, A. Postma, P. J. Pigram and L. Meagher, Biomaterials, 2016, 106, 24.
2 X. Wang, M. R. Salick, X. Wang, T. Cordie, W. Han, Y. Peng, Q. Li and L. S. Turng, Biomacromolecules, 2013, 14, 3557.

3 X. Qin, H. Zhang, J. Wu, X. Chu, Y. B. He, C. Han, C. Miao, S. Wang, B. Li and F. Kang, Carbon, 2015, 87, 347.

4 Q. Li, Y. Xu, H. Wei and X. Wang, RSC Adv., 2016, 6, 65275.

5 T. A. Valente, D. M. Silva, P. S. Gomes, M. H. Fernandes, J. D. Santos and V. Sencadas, ACS Appl. Mater. Interfaces, 2016, 8, 3241.

6 W. Liu, J. Lipner, C. H. Moran, L. Feng, X. Li, S. Thomopoulos and Y. Xia, Adv. Mater., 2015, 27, 2583.

7 J. L. Sang, S. H. Oh, J. Liu, S. Soker, A. Atala and J. J. Yoo, Biomaterials, 2008, 29, 1422.

8 S. Kaur, R. Barhate, S. Sundarrajan, T. Matsuura and S. Ramakrishna, Desalination, 2011, 279, 201.

9 A. Arinstein, J. Polym. Sci., Part B: Polym. Phys., 2013, 51, 756. 10 C. Lim, E. Tan and S. Ng, Appl. Phys. Lett., 2008, 92, 141908.

11 D. Papkov, Y. Zou, M. N. Andalib, A. Goponenko, S. Z. Cheng and Y. A. Dzenis, ACS Nano, 2013, 7, 3324.

12 X. Zhang, R. Nakagawa, K. H. K. Chan and M. Kotaki, Macromolecules, 2012, 45, 5494.

13 Y. Ding, P. Zhang, Z. Long, Y. Jiang, F. Xu and W. Di, J. Membr. Sci., 2009, 329, 56.

14 Z. M. Huang, Y. Z. Zhang, S. Ramakrishna and C. T. Lim, Polymer, 2004, 45, 5361.

15 T. J. Sill and H. A. von Recum, Biomaterials, 2008, 29, 1989. 16 L. Yan and Y. Lü, Eur. Polym. J., 2006, 42, 1696.

17 L. Huang, S. S. Manickam and J. R. Mccutcheon, J. Membr. Sci., 2013, 436, 213.

18 Z. Ma, M. Kotaki and S. Ramakrishna, J. Membr. Sci., 2006, 272, 179.

19 R. Sen, B. Zhao, D. Perea, M. E. Itkis, H. Hu, J. Love, A. Elena Bekyarova and R. C. Haddon, Nano Lett., 2004, 4, 459.

20 J. J. Mack, L. M. Viculis, A. Ali, R. Luoh, G. Yang, H. T. Hahn, F. K. Ko and R. B. Kaner, Adv. Mater., 2005, 17, 77.

21 Q. Iqbal, P. Bernstein, Y. Zhu, J. Rahamim, P. Cebe and C. Staii, Nanotechnology, 2015, 26, 105702.

22 R. Gopal, S. Kaur, Z. Ma, C. Chan, S. Ramakrishna and T. Matsuura, J. Membr. Sci., 2006, 281, 581.

23 M. Es-Saheb and A. Elzatahry, Int. J. Polym. Sci., 2014, 7, 1-6. 24 S. Moon and R. J. Farris, Polym. Eng. Sci., 2008, 48, 1848.

25 M. S. Cheal, K. J. Yeol and O. B. Taek, Polym. Eng. Sci., 2014, 54, 1289.

26 Y. Xu, Y. Gao, X. Wang, J. Jiang, J. Hou and Q. Li, Macromol. Mater. Eng., 2017, 302, 1700054.

27 K. Yoon, B. S. Hsiao and B. Chu, Polymer, 2009, 50, 2893.

28 P. Yu, X. Yao, Q. Han, S. Zang and Y. Gu, Polymer, 2014, 55, 6577. 